\title{
Modular Vehicle Architectures: A Systems Approach
}

\author{
Gary J. Rushton \\ Visteon Automotive Systems \\ Visteon Technical Center - Allen Park \\ 16630 Southfield Road \\ Allen Park, MI 48101 USA
}

\author{
Armen Zakarian \\ The University of Michigan - Dearborn \\ 2320 Engineering Complex \\ Dearborn, MI 48128 USA
}

\begin{abstract}
Modular systems provide the ability to achieve product variety through the combination and standardization of components. In this paper, a methodology that combines the Hatley/Pirbhai system model, integration analysis, and optimization techniques for development of modular electrical/ electronic vehicle systems is presented. The approach optimizes integration and interactions of the electrical/ electronic system elements and creates functional and physical modules for the vehicle. The paper illustrates importance of system modeling in developement of modular products. Discussion on how to make the system modeling more attractive to the industry is also presented.
\end{abstract}

\section{INTRODUCTION}

Modular systems provide the ability to achieve product variety through the combination and standardization of components. Potential benefits of modularity include (Ulrich 1991):

- Ecomomies of scale

- Increased ease of product/component change

- Increased product variety

- Reduced time to market

- Easier product diagnostics, maintenacnce, and repair.

To develop modular systems, well defined techniques and methodologies are required to assist the product development process. A classical product development process includes six phases shown in Figure 1.

This research focuses on the design phase of the product development process. Several studies have shown that product cost, quality, and time to market are most affected in the product design phase. For example, a study conducted at Ford Motor Company shows that only $5 \%$ of the manufacturing costs of a car are for design activities. However, the decisions made during the design phase affect $70 \%$ of the manufacturing costs. In a similar study, Xerox attributed $50 \%$ of the final product cost to the results of the design phase. Furthermore, $75 \%$ of the manufacturing costs are committed by the end of the preliminary design phase (Ullman 1992). These studies give a good indication of the impact of the design on the product cost.

This paper focuses on the modeling, analysis, and design of systems and illustrates the importance of system modeling in development of modular vehicle systems. The approach presented in this paper creates modular systems and optimizes integration and interactions of the system elements. The application of the approach is illustrated with an industrial example from the automotive industry, i.e., design of an instrument panel (cockpit). The Hatley/Pirbhai methodology is used for modeling functional requirements of the cockpit system of a vehicle. The Hatley/Pirbhai requirements model defines the interfaces (interactions) to support the functions of a cockpit system. Once the interfaces among the functions are identified, integration (cluster) analysis techniques are used to group the functions in the cockpit of a vehicle, and create electronic modules. The integration analysis approach uses analytical techniques to optimize interactions between system functions and group them in such a way that inter-cluster interactions are minimized (Pimmler 1994). A Hatley/Pirbhai architecture model is developed to represent the system design. The completed Hatley/Pirbhai system model, i.e., requirements and architecture, represents the functional requirements and the physical structure of the entire system. The overall system design process is shown in Figure 2.

\section{SYSTEM DESIGN}

In this section, different phases of the system design processes shown in Figure 2 are discussed.

Requirements Analysis. In this phase, the functional decomposition and a detailed system requirements model for the cockpit is developed. The Hatley/Pirbhai methodology is used for modeling the cockpit system. First, a top level system context diagram is constructed (see Figure 3). Once the cockpit system boundaries and external entities are established, the interactions between the cockpit system and the external entities are 
identified. The external entities in the context diagram are represented as terminators (rectangles).

The electrical/electronic functions of a cockpit system may be decomposed into six main functions (processes) shown in Figure 4:

1. Protect Occupants and Cargo

2. Provide Vehicle Motion

3. Provide Information, Communication, and Entertainment

4. Provide Visibility

5. Provide Interior Comfort

6. Control Power Distribution

All possible features of the cockpit system can be logically grouped into one of the six main processes presented above. Figure 4 is the child diagram of the context process shown in Figure 3 and presents the interactions among the six functions of the cockpit system. Each process in Figure 4 is further decomposed to the lowest level processes, i.e., primitives (PSPECs).

The functional decomposition and system modeling allows one to identify the functional elements of the system and their relationships. The system model represents the flow, processing, and transformation of data, energy, and material within the system. Once the Hatley/Pirbhai requirements model is constructed and the relationships between the functional elements (primitives) are identified, a function-function incidence (interaction) matrix for the cockpit system is developed (discussed next).

Integration Analysis. The Hatley/Pirbhai requirements model developed in the requirements analysis phase, identifies all the functional elements of the cockpit system and defines the interactions (interfaces) between the elements to support the functions of a cockpit system. Once the interactions among the functions are identified, a function-function interaction matrix of the interfaces is developed. A function-function interaction matrix $\left[\mathrm{a}_{\mathrm{ij}}\right]$ includes " 1 ", "blank" entries (see Figure 5), where an entry " 1 " indicates the information, material or energy link (signal flow) between functions $\mathrm{i}$ and $\mathrm{j}$, and the direction of the link (flow) is from $\mathrm{j} \rightarrow \mathrm{i}$.

A function-function interaction matrix in Figure 5 can be restructured using integration (cluster) analysis techniques to obtain the clusters in the binary interaction matrix and create functional modules (see Figure 6). Cluster analysis is concerned with the grouping of objects into homogeneous clusters (groups) based on the objects features. The application of cluster analysis techniques to the product design problem leads to the grouping of product functions and components (Kusiak 1998). In this research, the Extended Cluster Identification (ECI) algorithm is used to group the cockpit functions into modules (Kusiak 1987).
Improvement steps for the ECI algorithm is developed to maximize functional integration and minimize the interaction between the system functional modules. The application of the ECI algorithm and the improvement steps is explained next.

The ECI algorithm is an extension of the cluster identification algorithm (Iri 1968). The cluster identification algorithm identifies mutually separable clusters in the function-function interaction matrix. The ECI algorithm first checks for the existence of mutually separable clusters in the interaction matrix and if such a solution does not exist, heuristically solves the problem by randomly removing the bottleneck functions/interactions from the interaction matrix. A function (interaction) is considered a bottleneck, when it does not allow the decomposition of the functionfunction interaction matrix into mutually separable clusters.

In the modularity matrix $\left[\mathrm{a}_{\mathrm{ij}}\right]$, two types of bottleneck functions are possible: upper diagonal and lower diagonal (see Figure 7). Each bottleneck function, i.e., entry $\mathrm{x}_{\mathrm{ij}}=1$ in matrix $\left[\mathrm{a}_{\mathrm{ij}}\right]$ not belonging to any cluster (module) in the matrix $\left[\mathrm{a}_{\mathrm{ij}}\right]$, interacts with only two clusters (modules), i.e., $\mathrm{m}(1)$ and $\mathrm{m}(2)$ in matrix $\left[\mathrm{a}_{\mathrm{ij}}\right]$. The improvement steps developed in this research moves the bottleneck function (interaction) $\mathrm{x}_{\mathrm{ij}}$ $=1$ to module $\mathrm{m}(1)$ or $\mathrm{m}(2)$ if such an assignment improves the functional modularity of the system, i.e., maximizes interactions within the modules and minimizes interactions between the modules.

The two steps performed in the integration analysis phase are as follows:

1. Construct function - function interaction matrix

The Hatley/Pirbhai requirements model identifies the relationships between the functional elements, primitives. In essence, the model can be thought of as a huge network of primitive processes linked together by their flows (Hatley 1987). Once the system functions and their relationships are obtained, a function function interaction matrix for the system is constructed.

2. Construct modularity matrix

Apply the ECI algorithm and the improvement steps to obtain the modularity matrix.

Design Analysis. In this phase, the system design is completed. The Hatley/Pirbhai architecture model is developed to allocate the functional requirements and interactions from the Hatley/Pirbhai requirements model to the modules identified in the modularity matrix of cockpit system.

The application of the ECI algorithm and the improvement steps resulted in seven functional modules for the cockpit system (see Figures 8 and 9). The seven modules are shown in the cockpit architecture flow 
diagram (AFD) in Figure 8 and as follows:

1. Locking module

2. Climate Control module

3. Lighting module

4. Driver Information module

5. Entertainment module

6. Power pedal module

7. Ignition module

Each of the data flow lines shown in Figure 8 represent an interaction between functions contained within a module from the cockpit modularity matrix. For example, the entertainment module has an interaction with the locking module known as "RADIO PRESENT" and is represented as a data flow line in Figure 8.

Figure 9 shows the cockpit architecture interconnect diagram (AID). The AID represents the communication channels that exist between the cockpit architecture modules shown in Figure 8. The AID in Figure 9 has the same architecture modules as the AFD and shows the physical means by which the cockpit modules communicate (channels).

\section{DISCUSSION}

Today's systems are too complex to depend on the ability of a few overworked engineers to keep the various subsystems consistent. Systems have reached a point at which the interactions between the systems/subsystems are at least as complex as the subsystems themselves (Hatley 1987). Therefore, system models should be used to represent information that is gathered about the system in the requirements and design analysis phases.

The TurboCase/SYS software tool from Structsoft, Inc. was used for the development of the Hatley/Pirbhai requirements and architecture models. The TurboCase/SYS tool facilitates the allocation of functional elements in the requirements model to physical entities in the architecture model. System modeling tools can become even more attractive to the industry by integrating integration analysis techniques (i.e., ECI algorithm and improvement steps). The latter should allow the user, i.e., product developement engineers, to automatically determine the optimal number of modules and allocation of functions into each module.

\section{CONCLUSION}

This research developed a methodology that combined the system modeling, integration analysis, and optimization techniques for development of modular electrical/electronic systems. The approach optimized integration and interactions of the system elements and created functional and physical modules for the cockpit system.

The approaches presented in literature concentrate in partitioning simple products into physical modules without considering functional requirements of the system. This research demonstrated the importance of functional decomposition and system modeling of a complex system. The component analysis is very valuable at the detailed design phase. However, focusing on the functional analysis of the system allows one to develop several different system architecture and partitioning alternatives.

\section{REFERENCES}

Hatley, D. J. and Pirbhai, I. A. (1987), "Strategies for Real-Time Specification", Dorset House, New York.

Iri, M. (1968), "On the Synthesis of the Loop Cut Set Matrices and the Related Problem", RAAG Memoirs, Vol. 4, pp. 376-410.

Kusiak, A. and Chow, W. S. (1987), "Efficient Solving of the Group Technology Problem", Journal of Manufacturing Systems, Vol. 6, No. 2, pp. 117124.

Kusiak, A. (1998), "Group Technology”, University of Iowa, Working Paper Version 8-25.

Pimmler, T. U. and Eppinger, S. D. (1994), "Integration Analysis of Product Decomposition", Design Theory and Methodology - DTM, DE-Vol 68, ASME.

Ullman, D. G., (1992), “The Mechanical Design Process", McGraw-Hill, New York, NY.

Ulrich, K. and Tung, K. (1991), "Fundamentals of Product Modularity", DE-Vol. 39, Issues in Design Manufacture/Integration, ASME.

\section{BIOGRAPHY}

Gary Rushton has over 15 years of commercial and military electrical/electronic systems engineering experience. He has an MS in Automotive Systems Engineering from the University of Michigan. He is currently working as a systems engineering technical speicalist with Visteon Automotive Systems, an enterprise of Ford Motor Company. At Visteon he has worked on audio software, subsystem product development/design, diagnostics, vehicle system architectures and design, and cockpit systems design. Previously, with General Dynamics, he worked on avionics systems for the F-16 and vehicle electronics systems for the Abrams M1A2 tank. (grushton@visteon.com).

Armen Zakarian received his B.S. degree in mechanical engineering from Yerevan Polytechnic University, Yerevan, Armenia, his M.S. degree in 
industrial and systems engineering from the University of Southern California, Los Angeles, California and his Ph.D. degree in industrial engineering from The University of Iowa, Iowa City, Iowa, in 1997. He is an Assistant Professor of Industrial and Manufacturing Systems Engineering at the University of Michigan Dearborn. He taught at both undergraduate and graduate levels at The University of Iowa and has held a visiting faculty position at the American University of Armenia. He has published papers in jounals sponsored by IEEE and IIE societies. His research interests include development of products and systems, reliability and risk analysis of process models, and modeling and analysis of manufacturing systems. (zakarian@umich.edu).

Figure 1: Phases of Product Development Process

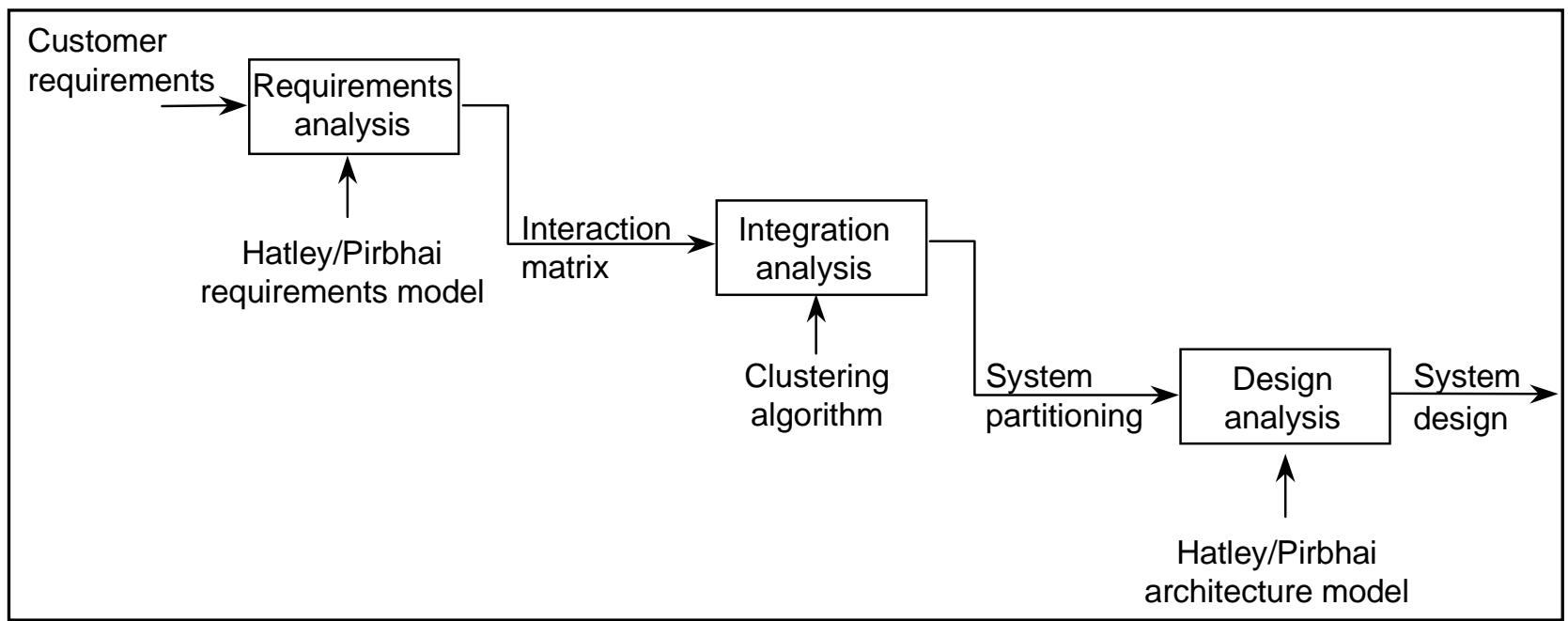

Figure 2: System Design Process 


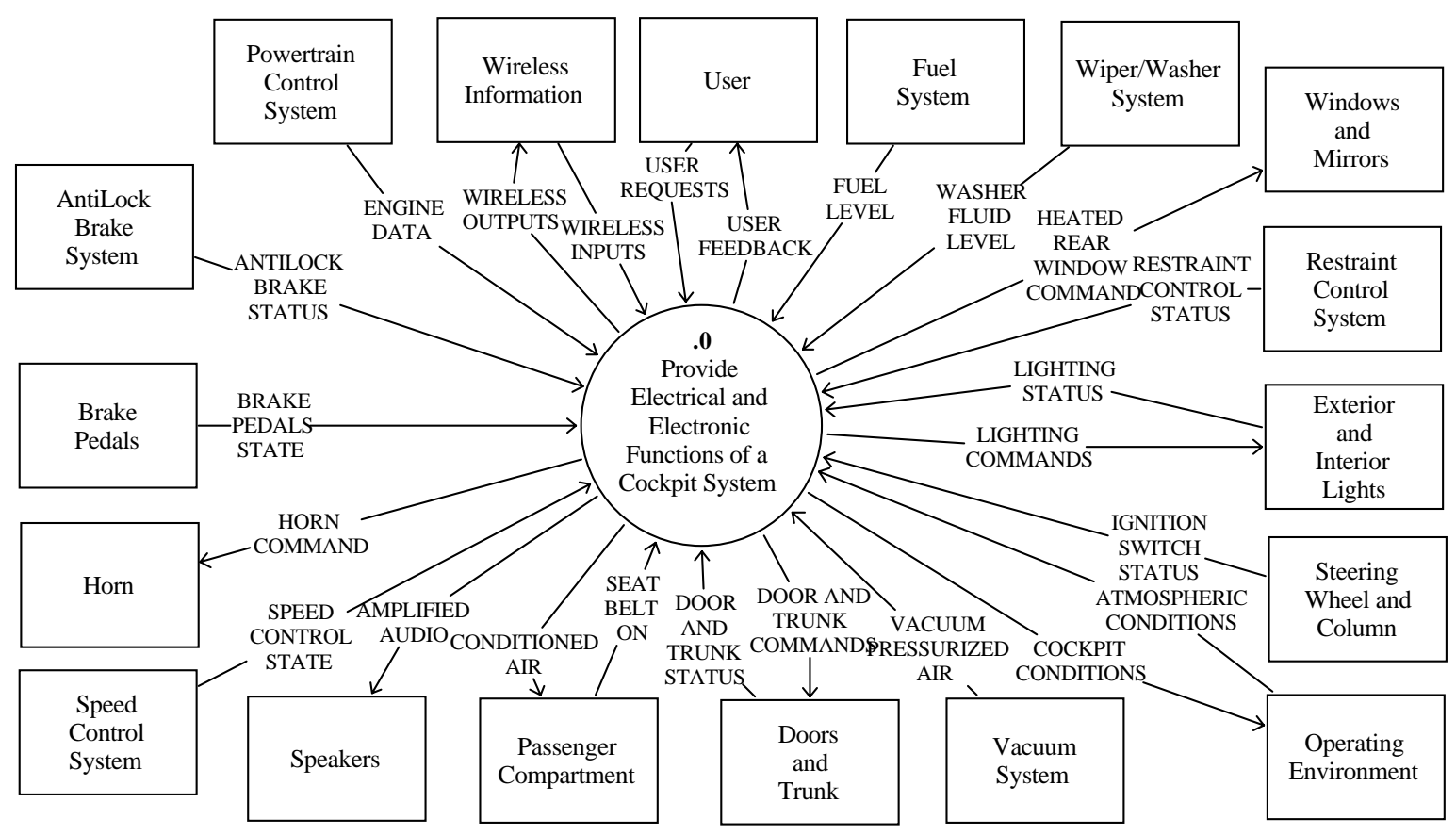

Figure 3: Cockpit System Context Diagram

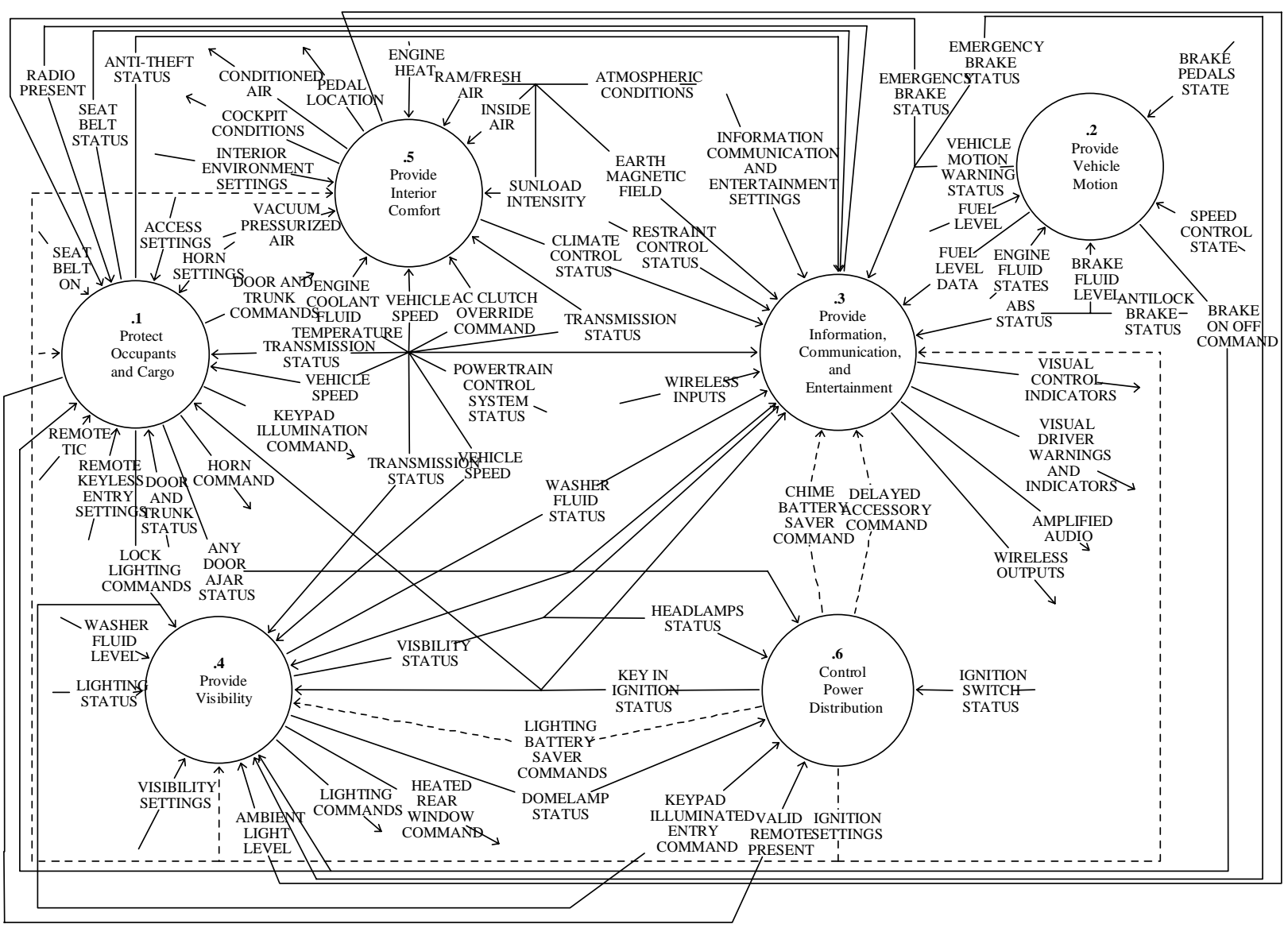

Figure 4: Provide Electrical/Electronic Functions of a Cockpit System 


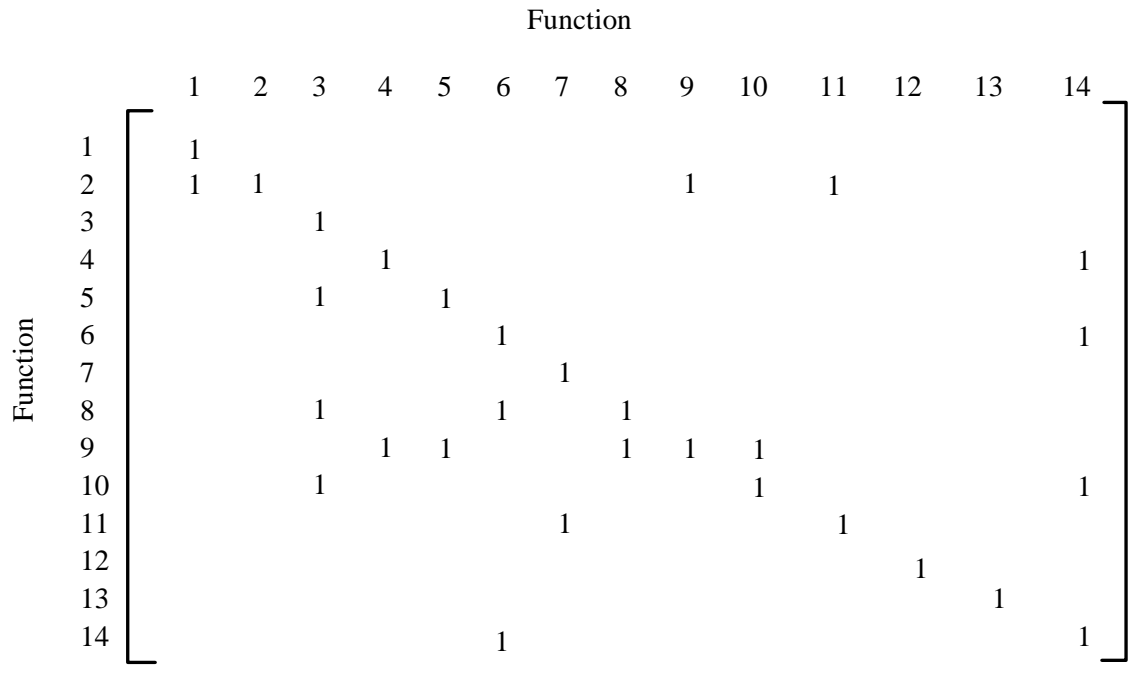

Figure 5: Function- Function Interaction Matrix

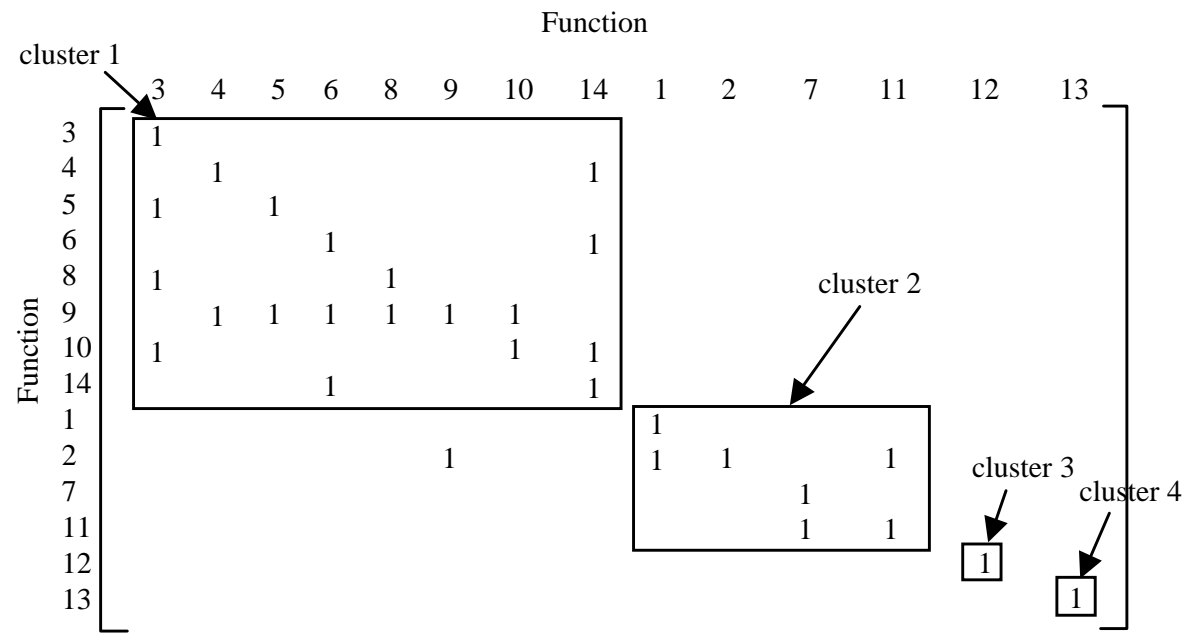

Figure 6: Modularity Matrix

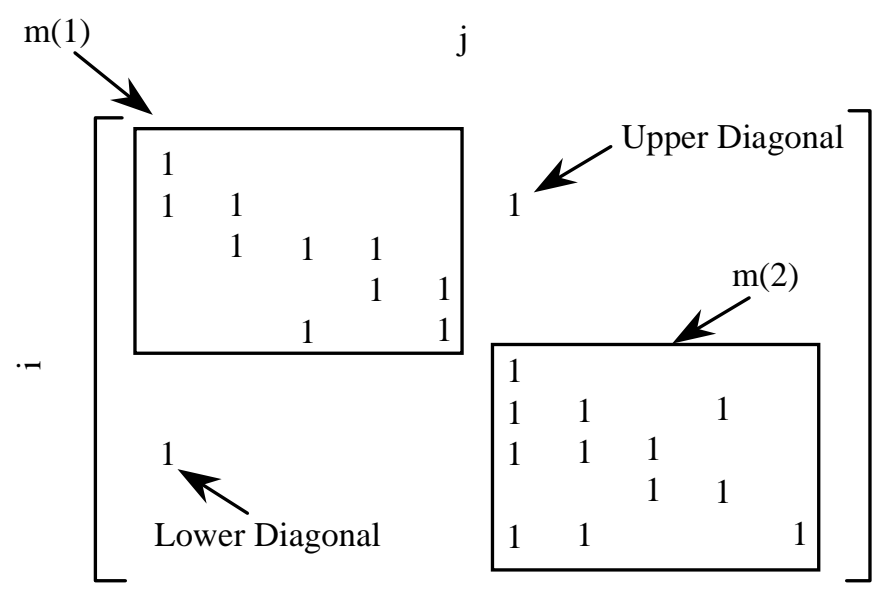

Figure 7: Upper/Lower Diagonal Bottleneck Functions 


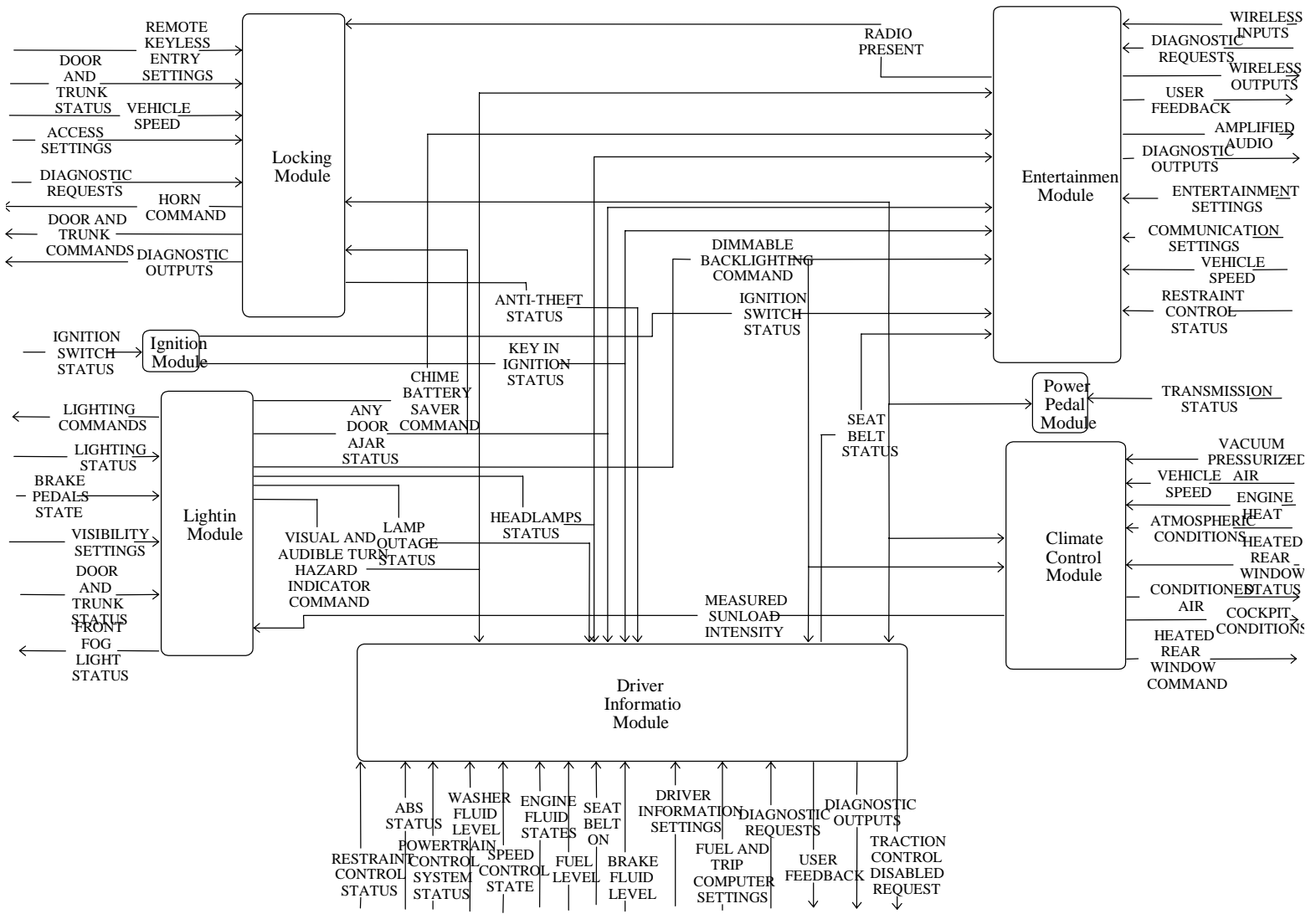

Figure 8: Cockpit Architecture Flow Diagram

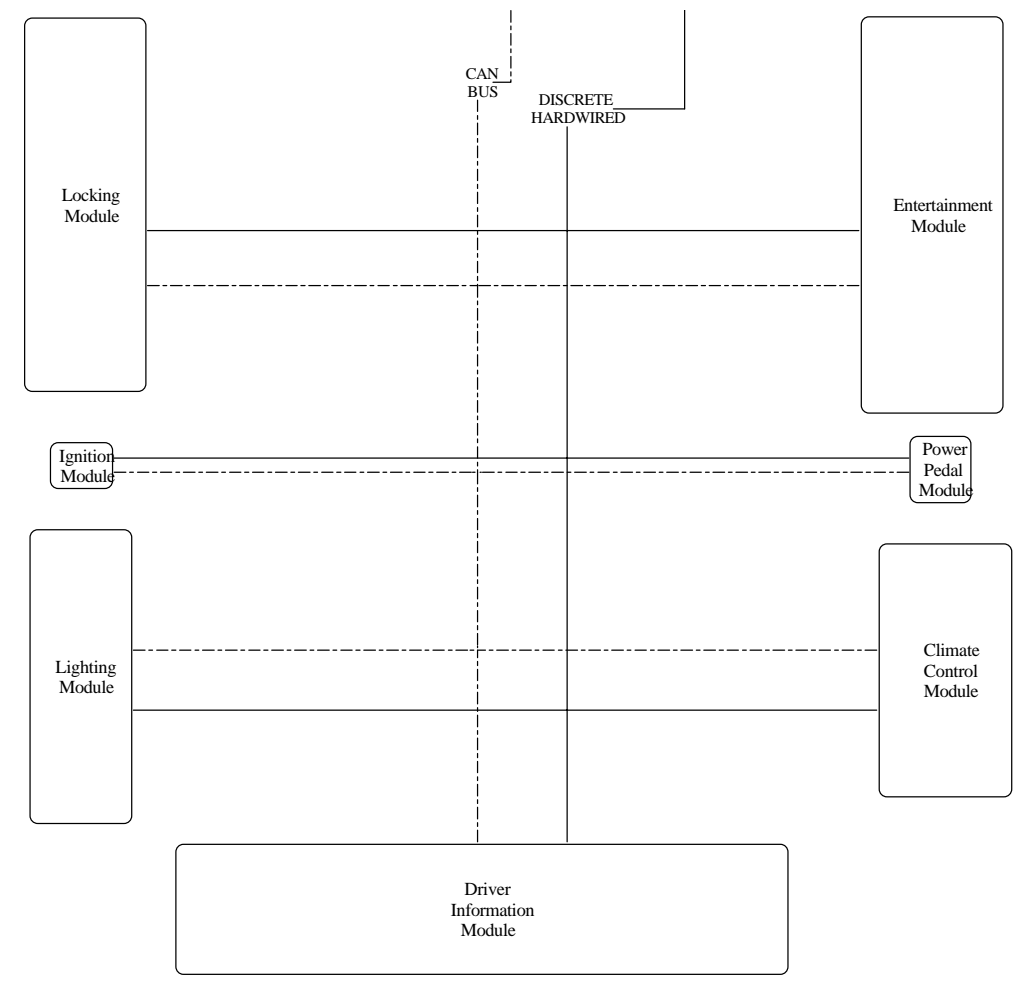

Figure 9: Cockpit Architecture Interconnect Diagram 